\title{
Colorimetric Detection of Mercury Species Based on Functionalized Gold Nanoparticles
}

\author{
Ling Chen, ${ }^{\dagger \neq, \S}$ Jinhua $\mathrm{Li}^{\dagger}{ }^{\dagger}$ and Lingxin Chen ${ }^{*}{ }^{\dagger}$ \\ ${ }^{\dagger}$ Key Laboratory of Coastal Environmental Processes and Ecological Remediation, Yantai Institute of Coastal Zone Research, Chinese \\ Academy of Sciences, Yantai 264003, China \\ ${ }^{\ddagger}$ School of Materials Science and Engineering, University of Jinan, Jinan 250022, China \\ ${ }^{\S}$ University of Chinese Academy of Sciences, Beijing 100049, China
}

\section{Supporting Information}

\begin{abstract}
The speciation analysis of heavy metal pollutants is very important because different species induce different toxicological effects. Nanomaterial-assisted optical sensors have achieved rapid developments, displaying wide applications to heavy metal ions but few to metal speciation analysis. In this work, a novel colorimetric nanosensor strategy for mercury speciation was proposed for the first time, based on the analyte-induced aggregation of gold nanoparticles (Au NPs) with the assistance of a thiol-containing ligand of diethyldithiocarbamate (DDTC). Upon the addition of mercury species, because $\mathrm{Hg}$-DDTC was more stable than $\mathrm{Cu}-\mathrm{DDTC}$, a placedisplacement between $\mathrm{Hg}$ species and $\mathrm{Cu}^{2+}$ would occur, and thereby the functionalized $\mathrm{Au}$ NPs would aggregate, resulting in a color change. Moreover, by virtue of the masking effect of ethylenediaminetetraacetic acid (EDTA), the nanosensor could readily discriminate organic mercury and inorganic mercury $\left(\mathrm{Hg}^{2+}\right)$, and it is thus anticipated to shed some light on the colorimetric sensing of organic mercury. So, a direct, simple

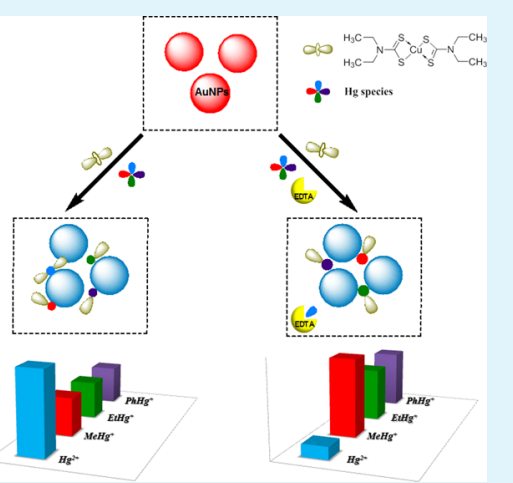
colorimetric assay for selective determination of $\mathrm{Hg}$ species was obtained, presenting high detectability, such as up to $10 \mathrm{nM}$ for $\mathrm{Hg}^{2+}$ and $15 \mathrm{nM}$ for methylmercury. Meanwhile, the strategy offered excellent selectivity toward mercury species against other metal ions. The simple, rapid, and sensitive label-free colorimetric sensor for the determination of $\mathrm{Hg}$ species provided an attractive alternative to conventional methods, which usually involve sophisticated instruments, complicated processes, and long periods of time. More importantly, by using mercury as a model, an excellent nanomaterial-based optical sensing platform can be developed for speciation analysis of trace heavy metals, which can lead to nanomaterials stability change through smart functionalization and reasonable interactions.
\end{abstract}

KEYWORDS: mercury speciation analysis, colorimetric detection, gold nanoparticles, aggregation

\section{INTRODUCTION}

Heavy metal pollutants exhibit different species and different toxicological effects, and therefore their speciation analysis is vital. Toxic mercury species are one of the significant widespread pollution sources. Organic forms of mercury $(\mathrm{Hg})$, such as methylmercury $(\mathrm{MeHg})$, ethylmercury $(\mathrm{EtHg})$ and phenylmercury $(\mathrm{PhHg})$, have much higher toxicity than inorganic and elemental $\mathrm{Hg}$ due to their high liposolubility and biomagnification factor (up to $10^{6}$ ) in the food chain. ${ }^{1}$ For instance, $\mathrm{MeHg}$ species readily cross the blood-brain barrier, accumulate in the brain, and cause damage to the central nervous system and other organs. ${ }^{2,3}$ It is generally accepted that all of the inorganic mercury species released in the ecosystem undergo biogeochemical transformation processes and can be converted into organic forms by microorganisms and microalgae in aquatic environments. ${ }^{4}$ In this case, recognition and detection of organic $\mathrm{Hg}$ is of utmost importance compared to measuring the total $\mathrm{Hg}$.

Methods currently available for detecting mercury species mainly include atomic absorption spectrometry, atomic fluorescence spectrometry, and inductively coupled plasma mass spectrometry. ${ }^{5-8}$ In general, these element-specific detectors are typically coupled with gas chromatography, high performance liquid chromatography, and capillary electrophoresis. ${ }^{9}$ However, these instrument techniques are generally rather complicated, costly, and time-consuming. Also, these conventional methods mostly cannot discriminate organic mercury from inorganic mercury and instead measure the total $\mathrm{Hg}$. Consequently, the development of rapid, simple, costeffective, selective, and sensitive methods and techniques for the determination of organic mercury at trace/ultratrace levels has become particularly significant but has proven to be a challenging task.

Recently, nanomaterial-based colorimetric assay has been widely used for rapid, specific, cost-effective, and robust analysis in environmental and biological samples, which allow trans-

Received: June 4, 2014

Accepted: August 25, 2014

Published: August 25, 2014 
forming target sensing events into color changes, readily seen by the naked-eye. ${ }^{10,11}$ In response to the challenging task for mercury detection, a variety of colorimetric nanosensors have flourished. $^{12-20}$ Those based on the typical DNA aptamer ${ }^{13-15}$ or specific ligands, ${ }^{16-18}$ functionalized nanomaterials, and the nanosurface oxidation reduction reaction ${ }^{19,20}$ have become more and more attractive due to their simplicity, high sensitivity, and selectivity. However, most of them mainly focus on sensing inorganic mercury ions $\left(\mathrm{Hg}^{2+}\right)$ and those for sensing of organic mercury are rare. To the best of our knowledge, there are few reports on the applications of colorimetric sensors for the metal speciation analysis, ${ }^{21,22}$ especially because of the lack of selective recognition ligands.

So, herein, we develop a simple, rapid, and sensitive colorimetric nanosensor for the determination of $\mathrm{Hg}$ species by using the interparticle plasmon coupling on analyte-induced aggregation of gold nanoparticles (Au NPs) with the assistance of specific recognition ligands. Because $\mathrm{Hg}$ has a high affinity for soft donors such as sulfur, a thiol-containing ligand, diethyldithiocarbamate (DDTC) was employed. The response to $\mathrm{Hg}$ species was mainly based on the displacement of copper ions $\left(\mathrm{Cu}^{2+}\right)$ by $\mathrm{Hg}$ in the Bis DDTC copper(II) complex $\left(\mathrm{CuDDTC}_{2}\right)$ with the formation of more stable $\mathrm{Hg}$-DDTC complex. The presented Au NP-based colorimetric nanosensor was demonstrated to discriminate organic mercury and inorganic mercury $\left(\mathrm{Hg}^{2+}\right)$ using ethylenediaminetetraacetic acid (EDTA) as a masking agent. As far as we are aware, this is the first demonstration for the simple direct analysis of several mercury species by colorimetric assay.

\section{EXPERIMENTAL SECTION}

Chemicals and Reagents. Hydrogen tetrachloroaurate (III) tetrahydrate $\left(\mathrm{HAuCl}_{4} \cdot 4 \mathrm{H}_{2} \mathrm{O}\right)$, trisodium citrate, diethyldithiocarbamate (DDTC) and ethylenediaminetetraacetic acid (EDTA) were received from Sinopharm Chemical Reagent Co., Ltd., China. $\mathrm{CuSO}_{4}$, $\mathrm{Cd}\left(\mathrm{NO}_{3}\right)_{2}, \mathrm{MgSO}_{4}, \mathrm{Zn}\left(\mathrm{NO}_{3}\right)_{2}, \mathrm{CoCl}_{2} \cdot 6 \mathrm{H}_{2} \mathrm{O}, \mathrm{Pb}\left(\mathrm{NO}_{3}\right)_{2}, \mathrm{MnSO}_{4}$, $\mathrm{FeCl}_{2}, \mathrm{BaCl}_{2}, \mathrm{AgNO}_{3}$, and so on were purchased from Aladdin. The standards of $\mathrm{HgCl}_{2}$, methylmercury $\left(\mathrm{MeHg}^{+}\right)$, ethylmercury $\left(\mathrm{EtHg}^{+}\right)$, and phenylmercury $\left(\mathrm{PhHg}^{+}\right)$were obtained from the CRM/RM Information Center of China. All the standards and stock solutions were stored at $4{ }^{\circ} \mathrm{C}$ in a refrigerator until use. All of the chemicals and reagents were of analytical grade and used without further purification, and deionized ultrapure water was used for aqueous solutions preparation in all of the experiments.

Instrumentation. Deionized water $(18.2 \mathrm{M} \Omega \mathrm{cm}$ specific resistances) was purified by a Cascada LS Ultrapure water purification system (Pall Corporation, Port Washington, NY ). UV-vis absorption spectra were measured on a Thermo Scientific NanoDrop 2000/ 2000C spectrophotometer (Thermo Fisher Scientific, Waltham, MA). Transmission electron microscopy (TEM) analysis was performed on a JEM-1230 electron microscope (JEOL, Ltd., Japan) operating at 100 $\mathrm{kV}$. X-ray photoelectron spectroscopy (XPS) was performed using an AXIS Ultra DLD instrument with $\mathrm{Mg} \mathrm{K} \alpha$ radiation as the X-ray source. All glassware used in the following procedure was cleaned in a bath of freshly prepared 3:1 $(\mathrm{v} / \mathrm{v}) \mathrm{HCl}-\mathrm{HNO}_{3}$, rinsed thoroughly in deionized water and dried in air.

Preparation of Au NPs. Au NPs were synthesized by the citratemediated reduction of $\mathrm{HAuCl}_{4}$ following previously published procedures $^{23,24}$ with necessary modifications. After an aqueous solution of $1 \mathrm{mM} \mathrm{HAuCl}$ (100 mL) was heated to reflux with vigorous stirring in a round-bottom flask fitted with a reflux condenser, $38.8 \mathrm{mM}$ sodium citrate $(10 \mathrm{~mL})$ was immediately added to the boiled solution. After the mixture boiled for another $30 \mathrm{~min}$ and become wine red, the heating was stopped. The obtained solution was cooled to room temperature and then stored at $4{ }^{\circ} \mathrm{C}$. The average size of nanoparticles identified by TEM was about $13 \mathrm{~nm}$. The particle concentration was measured by UV-vis spectroscopy and estimated to be about $9.7 \mathrm{nM}$ according to Lambert-Beer's Law by using the molar extinction coefficients at the wavelength of maximum absorption of 15 $\mathrm{nm}$ gold colloid as reported $\left[\varepsilon(15) 518 \mathrm{~nm}=3.6 \times 10^{8} \mathrm{~cm}^{-1} \mathrm{M}^{-1}\right]$.

Sensing of Mercury Species. For $\mathrm{Hg}^{2+}$ sensing, the ions were first added to the mixture of DDTC $(5 \mu \mathrm{L}, 0.5 \mathrm{mM})$ and $\mathrm{Cu}^{2+}(5 \mu \mathrm{L}$, $0.25 \mathrm{mM})$ in buffer solution $(890 \mu \mathrm{L})$, and then the Au NPs $(100 \mu \mathrm{L}$, 9.7 $\mathrm{nM}$ ) were added. The total volume of the mixture was approximately $1.0 \mathrm{~mL}$. After the mixture was incubated for several minutes, the UV-vis spectra were recorded. For organic forms of $\mathrm{Hg}$ detection, $\mathrm{MeHg}^{+}, \mathrm{EtHg}^{+}$and $\mathrm{PhHg}^{+}$were separately added to the mixture of $\mathrm{CuDDTC}_{2}$ complex and EDTA in buffer solution in the similar way as $\mathrm{Hg}^{2+}$ sensing.

Analysis of Real Water Samples. Drinking water samples were obtained from our institute. The water samples were spiked with standard $\mathrm{Hg}$ species at certain concentrations and then mixed with the stock solutions containing the Au NP probe and a PBS buffer solution ( $\mathrm{pH} 6.8)$.

\section{RESULTS AND DISCUSSION}

Proposed Mechanism of the CuDDTC Complex $_{2}$ Assisted Au-NP-Based Nanosensor for Hg Sensing. Scheme 1A illustrates the proposed sensing mechanism for $\mathrm{Hg}$ species. Originally, the $\mathrm{CuDDTC}_{2}$ complex was employed as a specific ligand to recognize the $\mathrm{Hg}$ species. Because $\mathrm{Hg}$ has a high affinity for soft donors such as sulfur, ${ }^{25,26}$ in the presence

Scheme 1. Schematic Illustrations of (A) Au NP-Based Colorimetric Strategy for $\mathrm{Hg}$ Species Sensing and (B) $\mathbf{H g}-$ DDTC Complex Attached to the Surface Of Au NPs

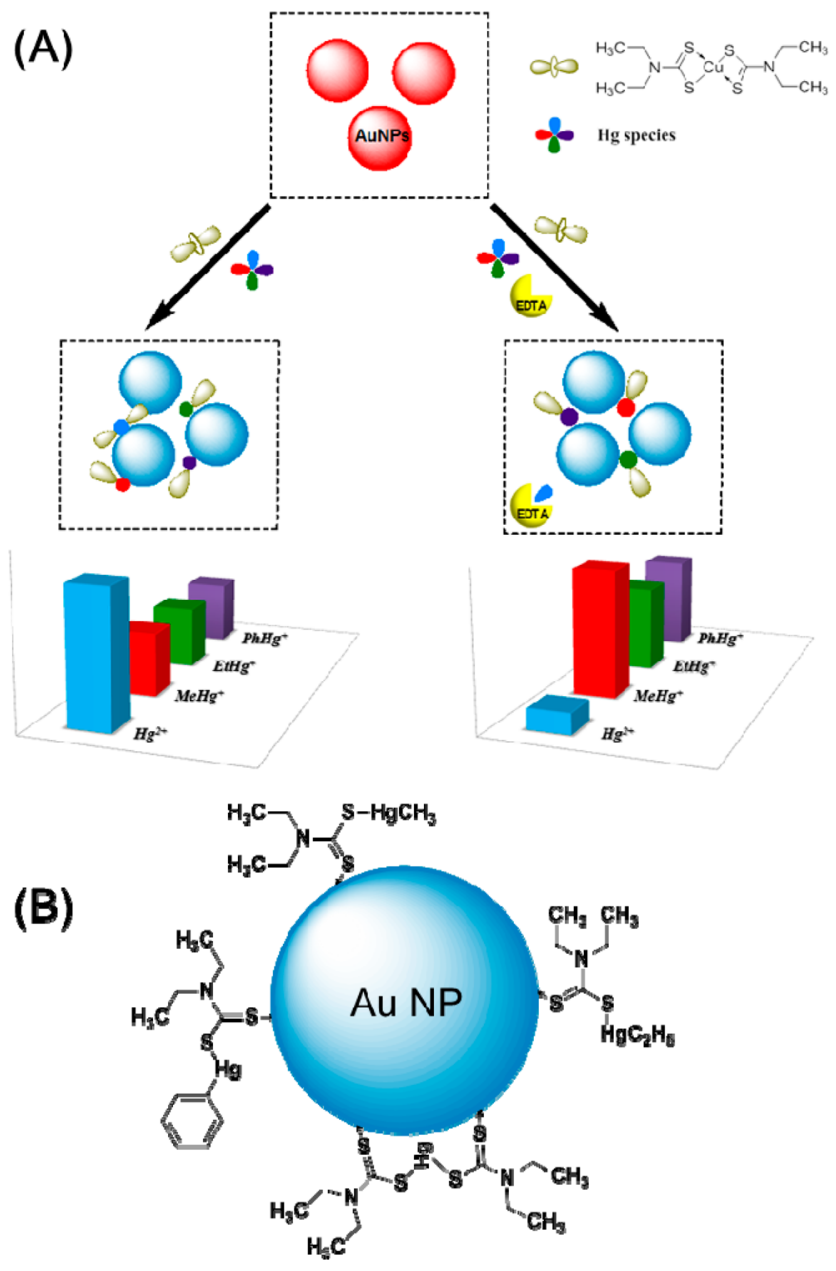




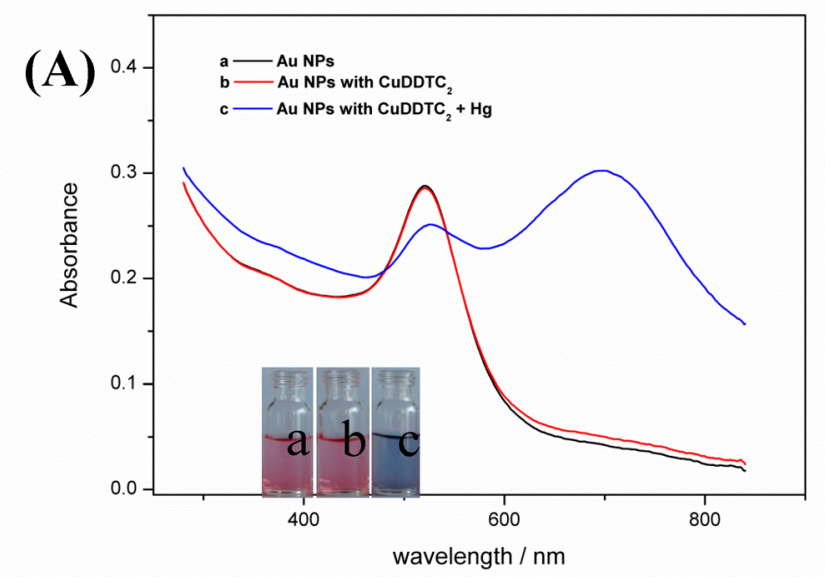

(B)

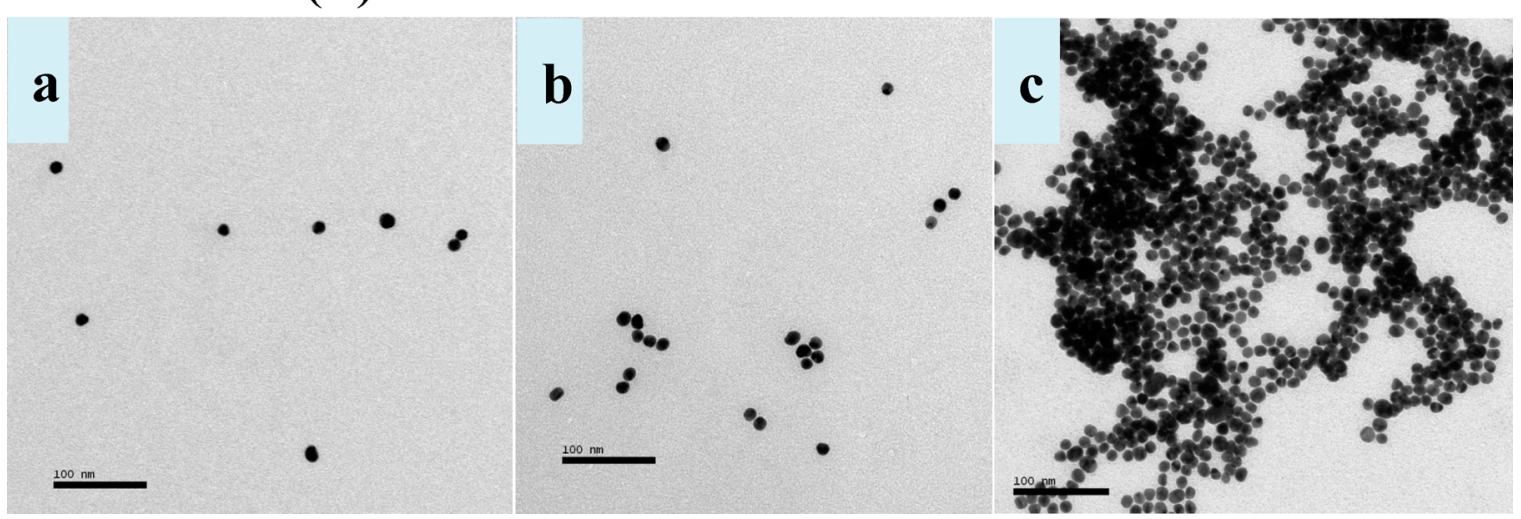

Figure 1. (A) UV-vis absorption spectra of Au NPs in the different conditions: (a) Au NPs, (b) Au NPs with CuDDTC 2 , and (c) Au NPs with $\mathrm{CuDDTC}_{2}+\mathrm{Hg}$ species. (A, inset) color changes. (B) TEM images corresponding to the Au NPs in panel A.

of $\mathrm{Hg}$ species, they can immediately displace the $\mathrm{Cu}^{2+}$ in the $\mathrm{CuDDTC}_{2}$ complex by promptly forming $\mathrm{Hg}-\mathrm{DDTC}$ complexes containing two residual thiol groups for $\mathrm{Hg}^{2+}$ and one for organic mercury $\left(\mathrm{MeHg}^{+}, \mathrm{EtHg}^{+}\right.$, and $\left.\mathrm{PhHg}^{+}\right)$, respectively, as shown in Formula S1 (Supporting Information). As is well-known, the citrate ions, which keep Au NPs from aggregation on the surfaces, can be easily displaced by thiol groups. ${ }^{20,27,28}$ Thus, it is supposed that the $\mathrm{Hg}$-DDTC complex could be attached to the surface of Au NPs through the $\mathrm{Au}-\mathrm{S}$ linkage, as shown in Scheme 1B. Consequently, the system became so destabilized and thereby resulted in the aggregation of $\mathrm{Au}$ NPs and a color change from red to blue, which is different from the reported aggregation induced by the deposition of $\mathrm{Hg}^{2+}$ onto the $\mathrm{Au} \mathrm{NP}$ surface. ${ }^{29}$ As shown in Figure 1A, a characteristic surface plasmon resonance (SPR) band of the prepared Au NPs was observed in the spectrum at approximately $520 \mathrm{~nm}$ (curve a, Figure 1A). With the addition of $\mathrm{CuDDTC}_{2}$ complex, the spectrum (curve b, Figure $1 \mathrm{~A}$ ) was pretty much the same as that of pure Au NPs (curve a, Figure 1A). Interestingly, upon addition of $\mathrm{Hg}$ species, the solution turned to blue along with the emergence of a long wavelength band at around $680 \mathrm{~nm}$ (curve c, Figure 1A). The inset images in Figure 1A show this color change from red to blue. The aggregation of Au NPs was further evidenced by TEM images that revealed monodispersed $\mathrm{Au}$ NPs in the absence of $\mathrm{Hg}$ and significant aggregation in the presence of $\mathrm{Hg}$, as seen in Figure 1B. Thus, the CuDDTC 2 -Au NPs system could carry out the colorimetric response to $\mathrm{Hg}^{2+}$ and organic mercury species with varying degrees (Scheme 1A). EDTA was introduced into the sensing system, which not only masked inorganic $\mathrm{Hg}^{2+}$, but also facilitated the sensing of organic mercury, as illustrated in Scheme 1A. Thus, this sensor also could readily discriminate inorganic mercury $\left(\mathrm{Hg}^{2+}\right)$ and organic mercury $\left(\mathrm{MeHg}^{+}\right.$, $\mathrm{EtHg}^{+}, \mathrm{PhHg}^{+}$) using a masking agent, and therefore, mercury speciation analysis could be easily realized.

To further investigate the reaction mechanism of this system for sensing mercury species, we added each species directly to the yellow $\mathrm{CuDDTC}_{2}$ solution and recorded the absorption spectra. As indicated in Figure 2, the formation of $\mathrm{Hg}$-DDTC complex could be explained by the evolution of the absorption spectra of $\mathrm{CuDDTC}_{2}$ complex upon the addition of $\mathrm{Hg}$. Figure 2 shows that the $\mathrm{CuDDTC}_{2}$ complex has a wide absorption spectrum with the peak at $460 \mathrm{~nm}$ (curve b), as compared to DDTC (curve a), due to the metal coordination effects of the conjugated system. Upon the addition of $\mathrm{Hg}$ species, the absorption band at $460 \mathrm{~nm}$ and the yellow color of the solution disappeared gradually, which indicated that the $\mathrm{Cu}^{2+}$ was displaced by $\mathrm{Hg}$ in the complex along with the formation of more stable $\mathrm{Hg}-\mathrm{DDTC}$ complex, because the mercury dithiocarbamate has a much higher equilibrium constant than copper dithiocarbamate. ${ }^{30-32}$ On account of the high affinity of $\mathrm{Hg}$ toward the thiol residual group, the resultant complexes of $\mathrm{Hg}^{2+}, \mathrm{MeHg}^{+}, \mathrm{EtHg}^{+}$, and $\mathrm{PhHg}^{+}$were estimated, respectively, as seen in Table S1 (Supporting Information). With the residual thiol group, the $\mathrm{Hg}-\mathrm{DDTC}$ complex could be attached to the surface of $\mathrm{Au}$ NPs through $\mathrm{Au}-\mathrm{S}$ bond 


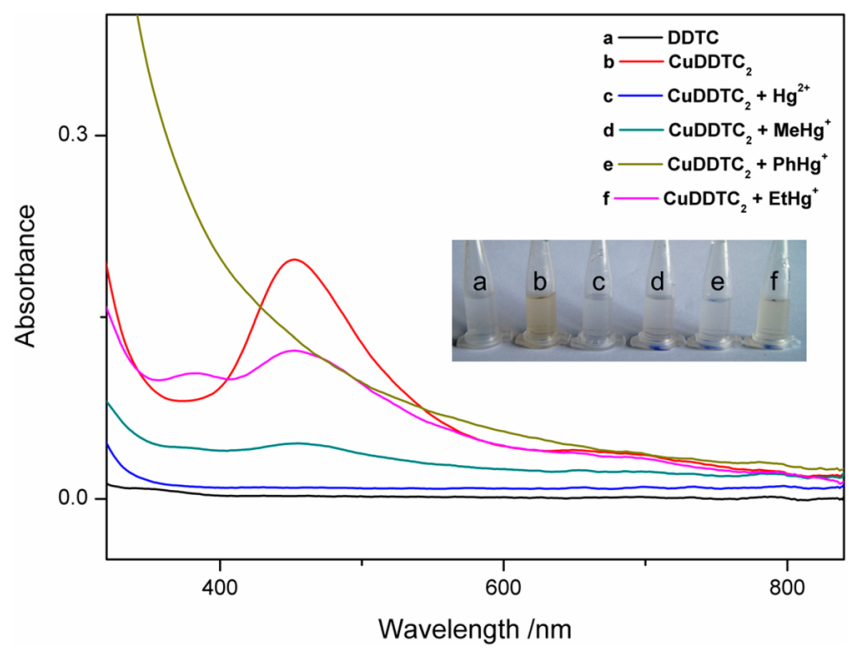

Figure 2. UV-vis absorption spectra of (a) DDTC $(30 \mu \mathrm{M})$ and (b) $\mathrm{CuDDTC}_{2}(15 \mu \mathrm{M})$ solutions upon the addition of $(\mathrm{c}) \mathrm{Hg}^{2+}(20 \mu \mathrm{M})$, (d) $\mathrm{MeHg}^{+}(20 \mu \mathrm{M}),(\mathrm{e}) \mathrm{PhHg}^{+}(20 \mu \mathrm{M})$, and (f) $\mathrm{EtHg}^{+}(10 \mu \mathrm{M})$, and (inset) corresponding photographic images of the solutions.

(Scheme 1B) and induce the aggregation of Au NPs. To better understand the interaction between $\mathrm{Hg}$ and $\mathrm{CuDDTC}_{2}$ complex, we freeze-centrifuged the mixture solutions of $\mathrm{Au}$ $\mathrm{NPs}$ and $\mathrm{CuDDTC}_{2}$ with the absence and presence of $\mathrm{Hg}^{2+}$, dried the solutions, and characterized them by XPS. As shown in Table S2 (Supporting Information), the atomic concentrations of $\mathrm{Hg}$ in the dried powders were measured to be about 0 and $0.97 \%$, respectively, indicating that all the four $\mathrm{Hg}$-DDTC complexes could be attached onto the surface of Au NPs. The results suggested that the $\mathrm{CuDDTC}_{2}$ complex could be readily transformed into a more stable $\mathrm{Hg}-\mathrm{DDTC}$ complex, which could induce the aggregation of $\mathrm{Au}$ NPs based on the $\mathrm{Au}-\mathrm{S}$ linkage.

Therefore, an $\mathrm{Au}$ NP-based colorimetric sensor for $\mathrm{Hg}$ species analysis was developed by utilizing interparticle plasmon coupling with analyte-induced aggregation of $\mathrm{Au}$ NPs, which resulted in a color change from red to blue. Moreover, with the aid of EDTA, only organic mercury could cause the aggregation of Au NPs. Hence, this colorimetric assay can be used to detect the presence of $\mathrm{Hg}$ species, especially delicately discriminate organic mercury species from $\mathrm{Hg}^{2+}$.

Optimization of Conditions. To investigate the optimized conditions in which Au NP-based colorimetric sensor can effectively detect $\mathrm{Hg}$ species, we tested the effects of $\mathrm{pH}$ titration, concentrations of DDTC and $\mathrm{Cu}^{2+}$, and reaction time. In Figure S1A (Supporting Information), the absorbance ratio (A680/A520) of Au NPs was low and constant when the solution (without $\mathrm{Hg}^{2+}$ ) $\mathrm{pH}$ ranged from 6.5 to 10.0, indicating that $\mathrm{CuDDTC}_{2}-\mathrm{Au}$ NPs were stable in the $\mathrm{pH}$ range. When the $\mathrm{pH}$ was lower than 6 , the absorbance ratio (A680/A520) of $\mathrm{CuDDTC}_{2}-\mathrm{Au} \mathrm{NPs}$ significantly increased. Under acidic conditions $(\mathrm{pH}<6.5)$, decomposition of $\mathrm{CuDDTC}_{2}$ complex resulted in the aggregation of Au NPs. The influence of $\mathrm{pH}$ on Hg-induced aggregation of $\mathrm{CuDDTC}_{2}-\mathrm{Au} \mathrm{NPs}$ was also shown in Figure S1A (Supporting Information); addition of $\mathrm{Hg}^{2+}$ resulted in a higher absorbance ratio (A680/A520). At $\mathrm{pH}$ $>9.0$, the absorbance ratio decreased because of the formation of colloidal $\mathrm{Hg}(\mathrm{OH})_{2} \cdot{ }^{31}$ Considering the decomposition of $\mathrm{CuDDTC}_{2}$ under acidic conditions, the optimal $\mathrm{pH}$ at 6.8 for detecting $\mathrm{Hg}$ was chosen in the following experiments. According to the changes of A680/A520, the concentrations of DDTC and $\mathrm{Cu}^{2+}$ were chosen at 2.0 and $1.0 \mu \mathrm{M}$, respectively (Figure S1B,C, Supporting Information). The reaction time was also studied under the optimized $\mathrm{pH}$ in the presence of $\mathrm{Hg}^{2+}(1.0 \mu \mathrm{M})$. The results showed that the maximum ratio of A680/A520 was obtained in $2 \mathrm{~min}$ after the addition of $\mathrm{Hg}^{2+}$ and remained unchanged with a further increase of reaction time, as shown in Figure S1D (Supporting Information), indicating that it was very fast to reach equilibrium in the interaction between $\mathrm{Hg}^{2+}$ and the $\mathrm{CuDDTC}_{2}-\mathrm{Au}$ NP-based sensor.

Feasibility for the Detection of $\mathrm{Hg}^{2+}$. The presented $\mathrm{Au}$ NP-based colorimetric sensor was first used to detect inorganic mercury, $\mathrm{Hg}^{2+}$, in the absence of EDTA. Figure $3 \mathrm{~A}$ shows the evolution of UV-vis spectra after adding different concentrations of $\mathrm{Hg}^{2+}$ to the sensor solution. With the increase of $\mathrm{Hg}^{2+}$ concentration from $10 \mathrm{nM}$ to $1.5 \mu \mathrm{M}$, the SPR band around $680 \mathrm{~nm}$ was gradually enhanced, demonstrating a sensitive response to $\mathrm{Hg}^{2+}$. Figure 3B shows the plot of aggregated degree (A680/A520) against the concentration of $\mathrm{Hg}^{2+}$. The fitted curve could be used for the accurate quantification of $\mathrm{Hg}^{2+}$ in a wide range of $10 \mathrm{nM}-1.5 \mu \mathrm{M}$, with a correlation coefficient of 0.997, as displayed in Figure $3 \mathrm{C}$, and the inset shows an excellent quantification of $\mathrm{Hg}^{2+}$ at low concentrations ranging from $10 \mathrm{nM}-0.1 \mu \mathrm{M}$. Moreover, the limit of detection (LOD, $3 \mathrm{~S} / \mathrm{N}$ ) could reach as low as 2.9 $\mathrm{nM}$, which meets the mandated upper limit of $10 \mathrm{nM}$ for $\mathrm{Hg}^{2+}$ in drinking water by U.S. Environmental Protection Agency (EPA).

The colorimetric nanosensor for the sensing response to other metal ions was investigated in detail. Figure S2 (Supporting Information) shows that the addition of 50-fold $\mathrm{Cd}^{2+}, \mathrm{Pb}^{2+}, \mathrm{Mg}^{2+}, \mathrm{Mn}^{2+}, \mathrm{Co}^{2+}, \mathrm{Ba}^{2+}, \mathrm{Zn}^{2+}$, and $\mathrm{Ag}^{+}$did not cause any obvious effect on the SPR band and the color of the colloid, while the organic forms of $\mathrm{Hg}$ at the same concentration $(0.8 \mu \mathrm{M})$ as $\mathrm{Hg}^{2+}$ caused significant change in the SPR band and color of the Au NPs. One main reason is that these metal ions have much poorer chelating ability with DDTC than $\mathrm{Hg}$ species and thus cannot replace the $\mathrm{Cu}^{2+}$ in the complex. $^{31,32}$ On the other hand, it was observed the three organic mercury species presented similar colorimetric responses, while $\mathrm{Hg}^{2+}$ afforded a much higher response. The difference of colorimetric response could be attributed to the effect of the alkyl group on the reaction activity. ${ }^{32,33}$ So, the nanosensor was demonstrated to be feasible for selectively detecting $\mathrm{Hg}^{2+}$.

EDTA Assistant Specific Detection of Organic Mercury. To further investigate this sensor for the determination of organic mercury, we introduced EDTA. EDTA containing rich $\mathrm{N}$ and $\mathrm{O}$ atoms is usually employed as metal-chelator and can be used as a masking agent to discriminate $\mathrm{Hg}^{2+}$ and $\mathrm{MeHg}^{+}$in typical methods. ${ }^{34}$ It is a great surprise that EDTA not only can mask inorganic $\mathrm{Hg}^{2+}$ but also can assist this method to recognize organic mercury, which endows the present colorimetric sensor an ability to discriminate $\mathrm{Hg}^{2+}$ and organic mercury. As shown in Figure 4, the addition of EDTA can effectively mask $\mathrm{Hg}^{2+}$ while accelerating the recognition of organic mercury. The effect of EDTA concentration on this nanosensor response was investigated, as shown in Figure S3A (Supporting Information). With the increase of EDTA concentration, $\mathrm{Hg}^{2+}$ was masked, and the response to organic mercury was effectively enhanced. It was supposed that the EDTA assisted the competition reaction between organic mercury and $\mathrm{CuDDTC}_{2}$ complex, $^{30}$ as indicated by the 

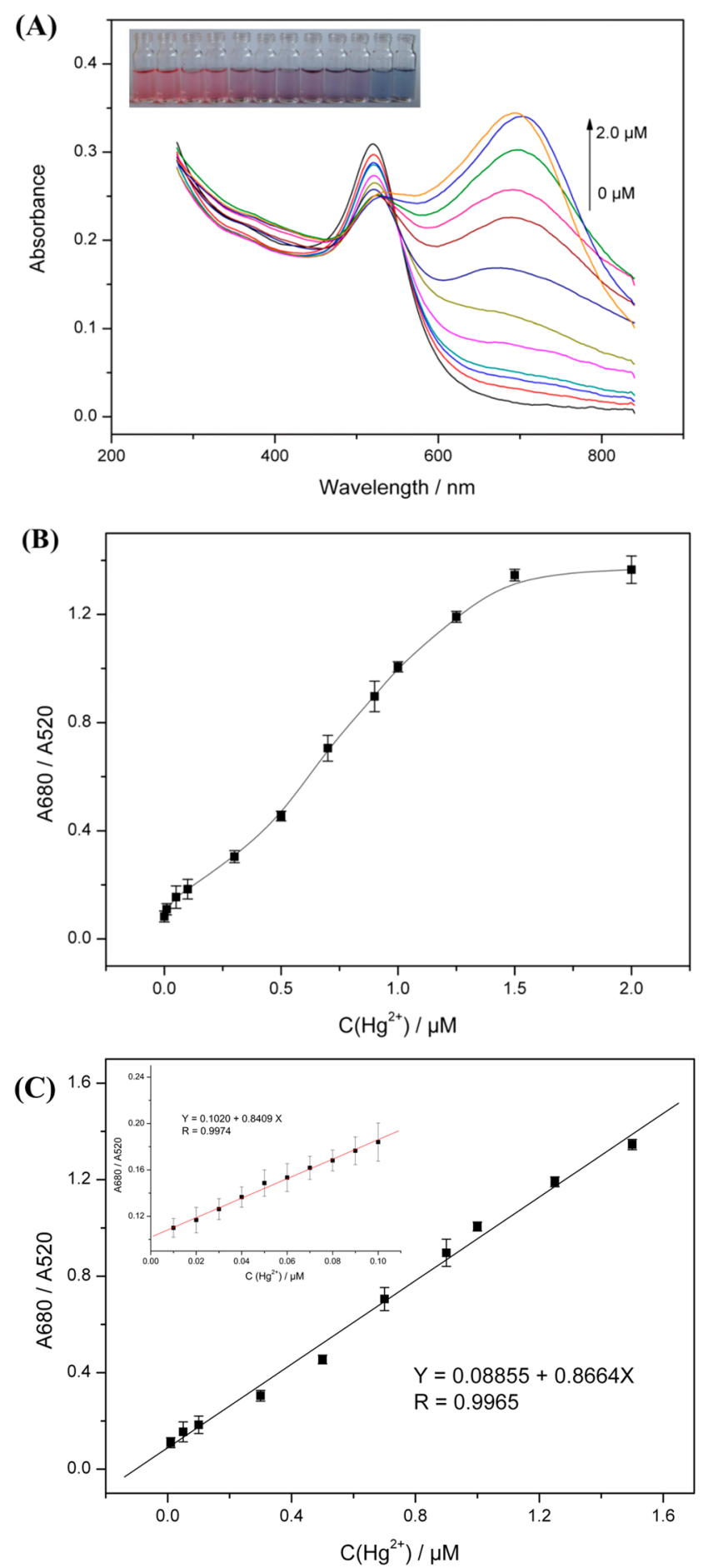

Figure 3. (A) UV-vis spectra of Au NPs sensing system with different concentrations of $\mathrm{Hg}^{2+}$ and (inset) colorimetric response to different concentrations of $\mathrm{Hg}^{2+}$. (B) The ratio values change of A680/A520 against different concentrations of $\mathrm{Hg}^{2+}$. (C) Linear curve in the range of 0.01 to $1.5 \mu \mathrm{M}$, and the inset shows a linear relationship ranging from 0.01 to $0.1 \mu \mathrm{M}$. The incubation time was $2 \mathrm{~min}$; the $\mathrm{pH}$ value of the solutions was 6.8; the error bars represent the standard deviations based on three independent measurements.

following reactions. Reaction 1 and Reaction 2 indicate that added excess EDTA can react with $\mathrm{CuDDTC}_{2}$ complex and thereby promote the displacement of $\mathrm{Cu}^{2+}$ by organic mercury, although EDTA has weaker affinity to organic mercury than

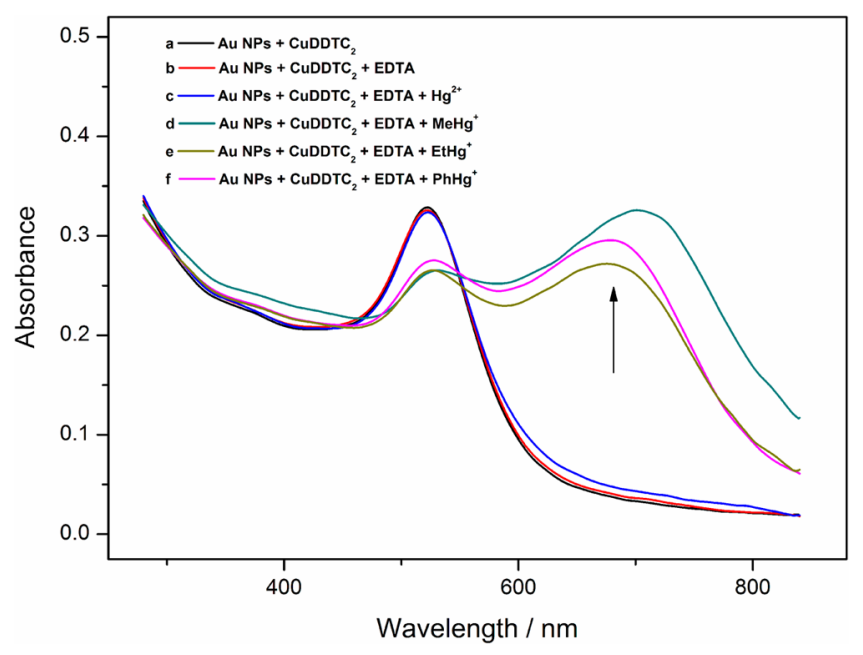

Figure 4. UV-vis spectra of Au NPs sensing system with different conditions: (a) Au NPs $+\mathrm{CuDDTC}_{2}(1.0 \mu \mathrm{M})$; (b) Au NPs + $\mathrm{CuDDTC}_{2}(1.0 \mu \mathrm{M})+\operatorname{EDTA}(0.1 \mathrm{mM}) ;(\mathrm{c}) \mathrm{Au} \mathrm{NPs}+\mathrm{CuDDTC}_{2}$ $(1.0 \mu \mathrm{M})+\operatorname{EDTA}(0.1 \mathrm{mM})+\mathrm{Hg}^{2+}(1.0 \mu \mathrm{M}) ;$ (d) Au NPs + $\mathrm{CuDDTC}_{2}(1.0 \mu \mathrm{M})+\operatorname{EDTA}(0.1 \mathrm{mM})+\mathrm{MeHg}^{+}(1.0 \mu \mathrm{M}) ;(\mathrm{e}) \mathrm{Au}$ $\mathrm{NPs}+\mathrm{CuDDTC}_{2}(1.0 \mu \mathrm{M})+\mathrm{EDTA}(0.1 \mathrm{mM})+\mathrm{EtHg}^{+}(1.0 \mu \mathrm{M})$; and (f) Au NPs $+\mathrm{CuDDTC}_{2}(1.0 \mu \mathrm{M})+$ EDTA $(0.1 \mathrm{mM})+\mathrm{PhHg}^{+}$ $(1.0 \mu \mathrm{M})$. The incubation time was $2 \mathrm{~min}$.

$\mathrm{Cu}^{2+}$. Finally, EDTA at $0.1 \mathrm{mM}$ was introduced in the sensing system.

$$
\begin{aligned}
& \mathrm{CuDDTC}_{2}+\mathrm{MeHg}^{+} \rightarrow \mathrm{MeHg}-\mathrm{DDTC}+\mathrm{Cu}^{2+} \\
& \mathrm{CuDDTC}_{2}+\mathrm{MeHg}^{+} \\
& \stackrel{\text { EDTA }}{\longrightarrow} \mathrm{MeHg}-\mathrm{DDTC}+\mathrm{Cu}-\text { EDTA } \\
& \quad \text { (Reaction 2) }
\end{aligned}
$$

The colorimetric response time of the $\mathrm{CuDDTC}_{2}-\mathrm{Au} \mathrm{NP}$ based probe to organic mercury was first investigated prior to the sensitivity study. As shown in Figure S3B (Supporting Information), the equilibrium was achieved in $2 \mathrm{~min}$, and thus 2 min was selected as the reaction time in the following tests for organic mercury. Figure 5 provides the responses to different organic mercury species $\left(\mathrm{MeHg}^{+}, \mathrm{EtHg}^{+}, \mathrm{PhHg}^{+}\right)$of this nanosensor, respectively. The ratio of A680/A520 was employed as the quantification indicator. Figure 5A,B shows the UV-vis spectra of $\mathrm{Au} \mathrm{NP}$-based nanosensor and response curve for the detection of different concentrations of $\mathrm{MeHg}^{+}$, and a good linear relationship between the ratio of A680/A520 and the $\mathrm{MeHg}^{+}$concentrations was obtained over the range of $0.015-0.8 \mu \mathrm{M}$ with an excellent quantification of $\mathrm{MeHg}^{+}$at low concentrations (Figure $5 \mathrm{~B}_{1}$ and inset). Figure $5 \mathrm{C}$,D displays the UV-vis spectra and the response change for different concentrations of $\mathrm{EtHg}^{+}$with a linear range of $0.026-1.3 \mu \mathrm{M}$ (Figure $5 \mathrm{D}_{1}$ ), and the response results of low concentrations were also shown in the inset of Figure $5 \mathrm{D}_{1}$. Figure $5 \mathrm{E}, \mathrm{F}$ depicts the $\mathrm{UV}$-vis spectra and response curve for $\mathrm{PhHg}^{+}$, presenting linearity within $0.15-1.20 \mu \mathrm{M}$ (Figure $5 \mathrm{~F}_{1}$ ). The corresponding LOD $(3 \mathrm{~S} / \mathrm{N})$ values were achieved up to $2.6,8.5$, and $30 \mathrm{nM}$ for $\mathrm{MeHg}^{+}, \mathrm{EtHg}^{+}$, and $\mathrm{PhHg}^{+}$, respectively. It demonstrated that the nanosensor could simply sensitively detect organic mercury species by using EDTA as masking agents.

Selectivity toward Organic Mercury and Practical Applications. To evaluate the selectivity of this colorimetric sensor in the presence of EDTA, we examined the competitive 
(A)

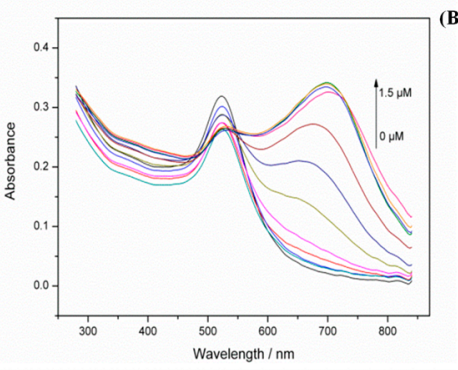

(C)

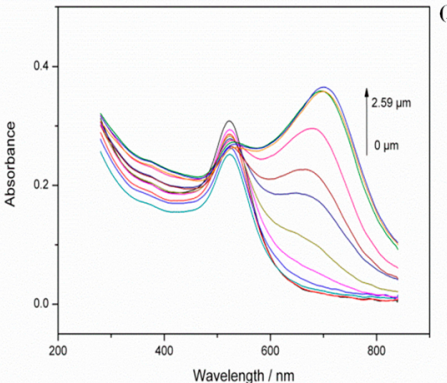

(E)

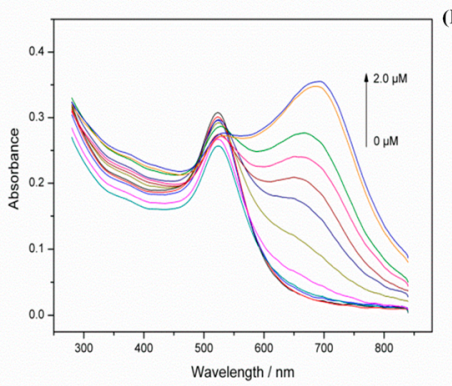

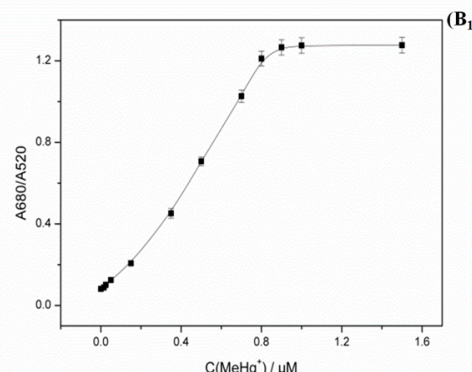
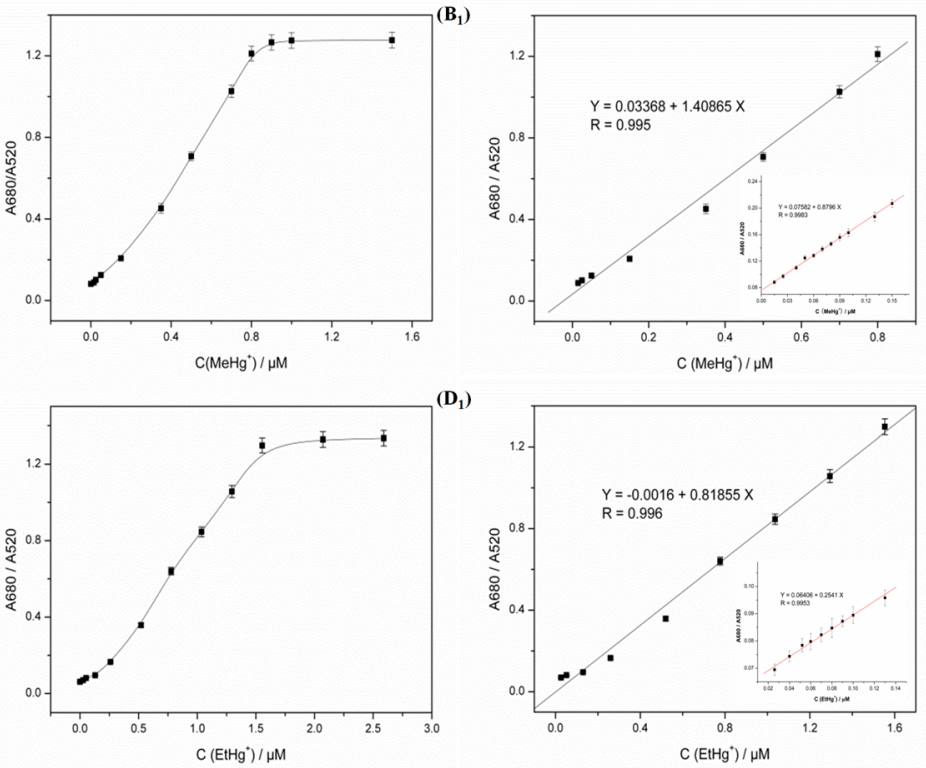

$\left(\mathbf{D}_{1}\right)$
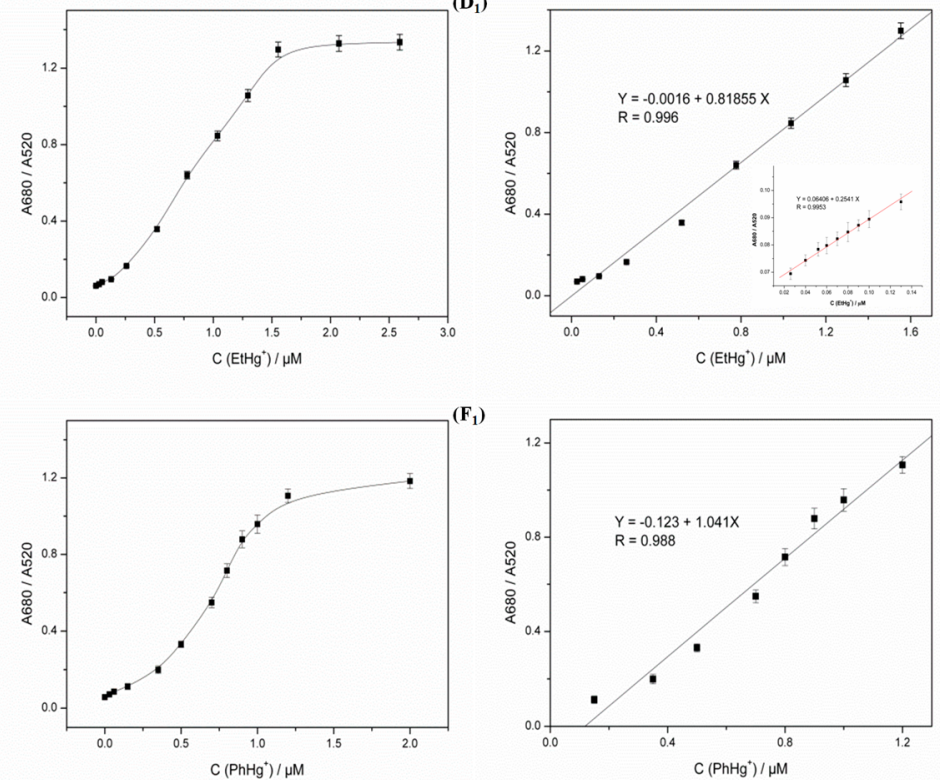

Figure 5. (A) UV-vis spectra of Au NPs sensing system with different concentrations of $\mathrm{MeHg}^{+}$; (B) ratio values change of A680/A520 against the concentration of $\mathrm{MeHg}^{+} ;\left(\mathrm{B}_{1}\right)$ linear plot over the range of $0.015-0.8 \mu \mathrm{M}$; (inset) linear relationship ranging from 0.015 to $0.13 \mu \mathrm{M}$. (C) $\mathrm{UV}-\mathrm{vis}$ spectra of Au NPs sensing system with different concentrations of EtHg ${ }^{+}$; (D) ratio values change of A680/A520 against the concentration of EtHg ${ }^{+}$; $\left(\mathrm{D}_{1}\right)$ linear plot over the range of $0.026-1.30 \mu \mathrm{M}$; (inset) linear relationship ranging from 0.026 to $0.15 \mu \mathrm{M}$. (E) UV-vis spectra of Au NPs sensing system with different concentrations of $\mathrm{PhHg}^{+} ;(\mathrm{F})$ ratio values change of $\mathrm{A} 680 / \mathrm{A} 520$ against the concentration of $\mathrm{PhHg}{ }^{+}$; $\left(\mathrm{F}_{1}\right)$ linear plot ranging from $0.15-1.20 \mu \mathrm{M}$. The error bars represent the standard deviations based on three independent measurements.

metal ions including $\mathrm{Cd}^{2+}, \mathrm{Pb}^{2+}, \mathrm{Mg}^{2+}, \mathrm{Mn}^{2+}, \mathrm{Co}^{2+}, \mathrm{Ba}^{2+}, \mathrm{Zn}^{2+}$, and $\mathrm{Ag}^{+}$under optimized conditions. The ratio of $\mathrm{A} 680 / \mathrm{A} 520$ and corresponding photographic images of the $\mathrm{Au} \mathrm{NP}$ system containing $0.8 \mu \mathrm{M}$ organic $\mathrm{Hg}$ species $\left(\mathrm{MeHg}^{+}, \mathrm{EtHg}^{+}\right.$, $\mathrm{PhHg}^{+}$), $8 \mu \mathrm{M} \mathrm{Hg}^{2+}$, and 50-fold other ions are vividly shown in Figure 6. As observed, the results demonstrated that only organic $\mathrm{Hg}$ species could cause the significant color change of the Au NPs, and 50-fold excess other metal ions had no obvious effect on the SPR band and the color of the colloid. Also, the $\mathrm{Hg}^{2+}$ was effectively masked by EDTA. The above results indicated that this developed method can effectively distinguish the inorganic mercury $\left(\mathrm{Hg}^{2+}\right)$ and organic mercury $\left(\mathrm{MeHg}^{+}, \mathrm{EtHg}^{+}, \mathrm{PhHg}^{+}\right)$with EDTA as the masking agent, while other metal ions had no disturbance. As seen in Figure 6, although the three organic mercury species could not be definitely distinguished and identified, $\mathrm{MeHg}^{+}$displayed significantly higher colorimetric response than $\mathrm{EtHg}^{+}$and $\mathrm{PhHg}^{+}$, which suggested EDTA also facilitated the displacement reaction and different alkyl groups had different effect on the reaction activity. ${ }^{30,31}$ More attempts and explorations should be made to effectively differentiate $\mathrm{MeHg}^{+}, \mathrm{PhHg}^{+}$, and $\mathrm{EtHg}^{+}$.

The developed nanosensor was further investigated for its practical applicability to organic mercury analysis. As shown in

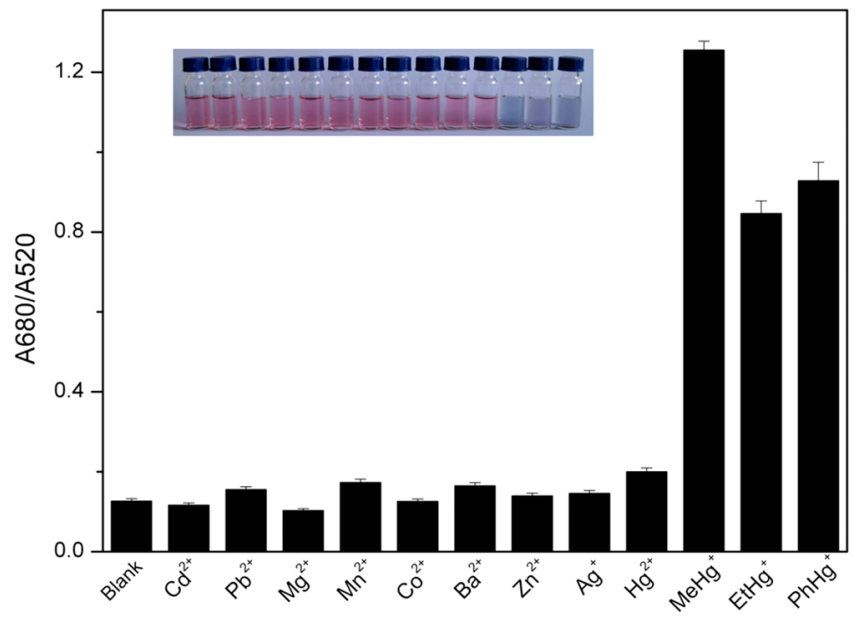

Figure 6. Ratio values of A680/A520 of Au NP-based sensing system with $\operatorname{EDTA}(0.1 \mathrm{mM})$ in the presence of $\mathrm{MeHg}^{+}(0.8 \mu \mathrm{M}), \mathrm{EtHg}^{+}(0.8$ $\mu \mathrm{M}), \mathrm{PhHg}^{+}(0.8 \mu \mathrm{M}), 10$-fold $\mathrm{Hg}^{2+}(8 \mu \mathrm{M})$, and 50 -fold other cation ions (individual $40 \mu \mathrm{M}$ ), respectively. (Inset, left to right) corresponding photographic images. The error bars represent the standard deviations based on three independent measurements. 
Table 1, satisfactory recoveries were obtained in a range of 93.3-102.4\% for two spiked concentration levels for the three

\begin{tabular}{|c|c|c|c|}
\hline & spiked (nM) & found $(\mathrm{nM})$ & recovery $(\%)$ \\
\hline \multirow[t]{3}{*}{$\mathrm{MeHg}^{+}$} & 0 & $\mathrm{nd}^{a}$ & \\
\hline & 100 & 96.0 & 96.0 \\
\hline & 300 & 307.2 & 102.4 \\
\hline \multirow[t]{3}{*}{$\mathrm{EtHg}^{+}$} & 0 & $\mathrm{nd}^{a}$ & \\
\hline & 300 & 289.1 & 96.4 \\
\hline & 500 & 492.3 & 98.5 \\
\hline \multirow[t]{3}{*}{$\mathrm{PhHg}^{+}$} & 0 & $\mathrm{nd}^{a}$ & \\
\hline & 450 & 437.2 & 97.2 \\
\hline & 600 & 559.6 & 93.3 \\
\hline
\end{tabular}

${ }^{a}$ Not detected.

organic mercury species in drinking water samples. The results clearly confirmed that this colorimetric nanosensor was potentially applicable for the accurate determination of organic mercury in real soft water samples. Meanwhile, it was found that the stability of the citrate protected Au NPs would be destroyed at high concentrations of cations (e.g., seawater matrix), causing the method to become invalid. Moreover, high contents of proteins and various other species (e.g., urine matrix) could easily lead to false results because they could also destruct the sensing system.

Recently, the Au NP-based nanosensors have emerged as international trends and presented excellent results and academic influences. Due to possible interference by undesired parameters on $\mathrm{Au} \mathrm{NPs,} \mathrm{especially} \mathrm{on} \mathrm{the} \mathrm{Au} \mathrm{NPs} \mathrm{without}$ particular ligand modification for recognizing a specific target, applying this type of colorimetric detection in complex samples has significant challenges. However, unmodified Au NP-based colorimetric methods have still been widely successfully developed; most of them have been stabilized with citrate or Tween coatings during the preparation of the nanoparticles, which have been proved effective for the detection of target ions/molecules with satisfying results. ${ }^{11,35}$ Therefore, although our method is presently suitable for soft natural waters rather than complex samples, inorganic mercury and organic mercury have indeed been effectively discriminated. Also, the method can be a potential candidate with suitable sample preparation.

\section{CONCLUSIONS}

In conclusion, a simple, rapid, and label-free colorimetric nanosensor with high selectivity and sensitivity was constructed on the basis of the analyte-induced aggregation of Au NPs and was successfully applied to speciation analysis of mercury. By taking advantage of the stronger interaction between $\mathrm{Hg}$ and DDTC, as well as the masking effect of EDTA, three organic mercury species could be easily discriminated from $\mathrm{Hg}^{2+}$. In contrast to conventional detection methods, a key architectural improvement is the simple colorimetric sensing of organic mercury based on Au NPs. The detection ability, such as 2.6 $\mathrm{nM}$ achieved for $\mathrm{MeHg}^{+}$, is comparable to or even surpasses that afforded by conventional methods. Besides, more efforts are expected to further identify and detect various organic mercury species. Because many nanomaterials and ligands, as well as specific interactions between targets and substrates, have been exploited and some of them are even commercially or readily available, such a sensing strategy can provide a simple, effective optical sensing platform for colorimetric analysis of heavy metal speciation and hold great potential for the fabrication of a number of optical sensors for various metal species based on target-induced nanomaterials stability changes.

As is well-known, the applicability of nanosensors in complex samples has always been the bottleneck of challenges and effort directions. So, the applications of novel optical nanosensors in complex sample analysis will also be continuously investigated and explored. Recently, we have developed a highly sensitive visual method for detecting $\mathrm{Cu}^{2+}$ based on the shape-dependent localized surface plasmon resonance spectroscopy of gold nanorods, and it successfully detected $\mathrm{Cu}^{2+}$ directly in a complex matrix, especially in seawater. ${ }^{36}$ This gives us some inspirations to develop colorimetric methods for the detection of organic mercury in complex samples. Also, we hope that the interesting attempt and new insights into the heavy metal speciation analysis will bring optimistic expectations for nanosensors that are applicable for both the heavy metal speciation analysis and complex samples. Accordingly, the successful implementation of this concept, that is, "functionalized optical nanosensor techniques for speciation analysis of trace heavy metals", will supply new principles and detection methodologies for heavy metal speciation analysis and enrich research contents of environmental/analytical chemistry, having both great scientific and practical values.

\section{ASSOCIATED CONTENT}

\section{S Supporting Information}

Structure formulas of mercury-DDTC and $\mathrm{Cu}-D D T C$ complexes, XPS data, sensing condition optimization data, selectivity test results, and the effects of EDTA concentration and reaction time. This material is available free of charge via the Internet at http://pubs.acs.org.

\section{AUTHOR INFORMATION}

\section{Corresponding Author}

*Tel./Fax: +86 535 2109130. E-mail: lxchen@yic.ac.cn.

\section{Notes}

The authors declare no competing financial interest.

\section{ACKNOWLEDGMENTS}

This work was financially supported by the National Natural Science Foundation of China (21275158), the Scientific Research Foundation for the Returned Overseas Chinese Scholars, the State Education Ministry, and the Innovation Projects of the Chinese Academy of Sciences (KZCX2-EW206).

\section{REFERENCES}

(1) Gao, Y.; Shi, Z. M.; Long, Z.; Wu, P.; Zheng, C. B.; Hou, X. D. Determination and Speciation of Mercury in Environmental and Biological Samples by Analytical Atomic Spectrometry. Microchem. J. 2012, 103, 1-14.

(2) Clarkson, T. W.; Magos, L. the Toxicology of Mercury and Its Chemical Compounds. Crit. Rev. Toxicol. 2006, 36 (8), 609-662.

(3) Kirka, J. L.; Lehnherrb, I.; Anderssonc, M.; Brauned, B. M.; Chane, L.; Dastoorf, A. P.; Durnfordg, D.; Gleasona, A. L.; Losetoh, L. L.; Steffeni, A.; Louisj, V. L. S. Mercury in Arctic Marine Ecosystems: Sources, Pathways, and Exposure. Environ. Res. 2012, 119, 64-87.

(4) Lehnherr, I.; Louis, V. L. S.; Hintelmann, H.; Kirk, J. L. Methylation of Inorganic Mercury in Polar Marine Waters. Nat. Geosci. 2011, 4, 298-302. 
(5) Leopold, K.; Foulkes, M.; Worsfold, P. Methods for the Determination and Speciation of Mercury in Natural Waters-A Review. Anal. Chim. Acta 2010, 663, 127-138.

(6) Segadea, S. R.; Tyson, J. F. Determination of Methylmercury and Inorganic Mercury in Water Samples by Slurry Sampling Cold Vapor Atomic Absorption Spectrometry in a Flow Injection System after Preconcentration on Silica C18 Modified. Talanta 2007, 71, 16961702.

(7) Leermakers, M.; Baeyens, W.; Quevauviller, P.; Horvat, M. Mercury in Environmental Samples: Speciation, Artifacts, and Validation. TrAC, Trends Anal. Chem. 2005, 24 (5), 383-393.

(8) Leopold, K.; Foulkes, M.; Worsfold, P. J. Preconcentration Techniques for the Determination of Mercury Species in Natural Waters. TrAC, Trends Anal. Chem. 2005, 28 (4), 426-435.

(9) Yang, F. F.; Li, J. H.; Lu, W. H.; Wen, Y. Y.; Cai, X. Q.; You, J. M.; Ma, J. P.; Ding, Y. J.; Chen, L. X. Speciation Analysis of Mercury in Water Samples by Dispersive Liquid-Liquid Microextraction Coupled to Capillary Electrophoresis. Electrophoresis 2014, 35, 474-481.

(10) Wang, Z. X.; Ma, L. Gold Nanoparticle Probes. Coord. Chem. Rev. 2009, 253, 1607-1618.

(11) Saha, K.; Agasti, S. S.; Kim, C.; Li, X. N.; Rotello, V. M. Gold Nanoparticles in Chemical and Biological Sensing. Chem. Rev. 2012, 112, 2739-2779.

(12) Du, J. J.; Jiang, L.; Shao, Q.; Liu, X. G.; Marks, R. S.; Ma, J.; Chen, X. D. Colorimetric Detection of Mercury Ions Based on Plasmonic Nanoparticles. Small 2013, 9 (9-10), 1467-1481.

(13) Lee, J. S.; Han, M. S.; Mirkin, C. A. Colorimetric Detection of Mercuric Ion $\left(\mathrm{Hg}^{2+}\right)$ in Aqueous Media using DNA-Functionalized Gold Nanoparticles. Angew. Chem., Int. Ed. 2007, 46, 4093-4096.

(14) Liu, C. W.; Hsieh, Y. T.; Huang, C. C.; Lin, Z. H.; Chang, H. T. Detection of Mercury(II) Based on $\mathrm{Hg}^{2+}$-DNA Complexes Inducing the Aggregation of Gold Nanoparticles. Chem. Commun. 2008, 19, $2242-2244$

(15) Xue, X. J.; Wang, F.; Liu, X. G. One-Step, Room Temperature, Colorimetric Detection of Mercury $\left(\mathrm{Hg}^{2+}\right)$ Using DNA/Nanoparticle Conjugates. J. Am. Chem. Soc. 2008, 130 (11), 3244-3245.

(16) Chen, L.; Lou, T. T.; Yu, C. W.; Kang, Q.; Chen, L. X. N-1-(2Mercaptoethyl)thymine Modification of Gold Nanoparticles: A Highly Selective and Sensitive Colorimetric Chemosensor for $\mathrm{Hg}^{2+}$. Analyst 2011, 136 (22), 4770-4773.

(17) Huang, C. C.; Chang, H. T. Parameters for Selective Colorimetric Sensing of Mercury(II) in Aqueous Solutions using Mercaptopropionic Acid-Modified Gold Nanoparticles. Chem. Commun. 2007, 12, 1215-1217.

(18) Lou, T. T.; Chen, L.; Zhang, C. R.; Kang, Q.; You, H. Y.; Shen, D. Z.; Chen, L. X. A Simple and Sensitive Colorimetric Method for Detection of Mercury Ions Based on Anti-Aggregation of Gold Nanoparticles. Anal. Methods 2012, 4 (2), 488-491.

(19) Rex, M.; Hernandez, F. E.; Campiglia, A. D. Pushing the Limits of Mercury Sensors with Gold Nanorods. Anal. Chem. 2006, 78 (2), 445-451.

(20) Lou, T. T.; Chen, Z. P.; Wang, Y. Q.; Chen, L. X. Blue-to-Red Colorimetric Sensing Strategy for $\mathrm{Hg}^{2+}$ and $\mathrm{Ag}^{+}$via Redox-Regulated Surface Chemistry of Gold Nanoparticles. ACS Appl. Mater. Interfaces 2011, 3 (5), 1568-1573.

(21) Ozay, O.; Ozay, H. Separation and Speciation of Iron Ions by Using Colorimetric Hydrogels. J. Macromol. Sci., Part A: Pure Appl.Chem. 2014, 51 (4), 308-317.

(22) Liu, Y.; Wang, X. X. Colorimetric Speciation of $\mathrm{Cr}(\mathrm{III})$ and $\mathrm{Cr}(\mathrm{VI})$ with a Gold Nanoparticle Probe. Anal. Methods 2013, 5 (6), $1442-1448$.

(23) Grabar, K. C.; Freeman, R. G.; Hommer, M. B.; Natan, M. J. Preparation and Characterization of Au Colloid Monolayers. Anal. Chem. 1995, 67 (4), 735-743.

(24) Haiss, W.; Thanh, N. T. K.; Aveyard, J.; Fernig, D. G. Determination of Size and Concentration of Gold Nanoparticles from UV-vis Spectra. Anal. Chem. 2007, 79 (11), 4215-4221.
(25) Nolan, E. M.; Lippard, S. J. A “Turn-On” Fluorescent Sensor for the Selective Detection of Mercuric Ion in Aqueous Media. J. Am. Chem. Soc. 2003, 125 (47), 14270-14271.

(26) Li, P. J.; Zhang, X.; Hu, B. Phase Transfer Membrane Supported Liquid-Liquid-Liquid Microextraction Combined with Large Volume Sample Injection Capillary Electrophoresis-Ultraviolet Detection for the Speciation of Inorganic and Organic Mercury. J. Chromatogr., A 2011, 1218, 9414-9421.

(27) Ai, K.; Liu, Y. L.; Lu, L. H. Hydrogen-Bonding RecognitionInduced Color Change of Gold Nanoparticles for Visual Detection of Melamine in Raw Milk and Infant Formula. J. Am. Chem. Soc. 2009, 131 (27), 9496-9497.

(28) Jiang, Y.; Zhao, H.; Zhu, N. N.; Lin, Y. Q.; Yu, P.; Mao, L. Q. A Simple Assay for Direct Colorimetric Visualization of Trinitrotoluene at Picomolar Levels Using Gold Nanoparticles. Angew. Chem., Int. Ed. 2008, 47, 8601-8604.

(29) Ojea-Jiménez, I.; López, X.; Arbiol, J.; Puntes, V. Citrate-Coated Gold Nanoparticles as Smart Scavengers for Mercury (II) Removal from Polluted Waters. ACS Nano 2012, 6 (3), 2253-2260.

(30) Fritz, J. S.; Sutton, S. A. Titration of Mercury with Bis(2hydroxyethyl)dithiocarbamate. Anal. Chem. 1956, 28 (8), 1300-1303.

(31) Yuan, C.; Liu, B. H.; Liu, F.; Han, M. Y.; Zhang, Z. P. Fluorescence "Turn On" Detection of Mercuric Ion Based on Bis(dithiocarbamato)copper(II) Complex Functionalized Carbon Nanodots. Anal. Chem. 2014, 86 (2), 1123-1130.

(32) Yan, X. P.; Li, Y.; Jiang, Y. Selective Measurement of Ultratrace Methylmercury in Fish by Flow Injection On-Line Microcolumn Displacement Sorption Preconcentration and Separation Coupled with Electrothermal Atomic Absorption Spectrometry. Anal. Chem. 2003, 75 (10), 2251-2255.

(33) Li, Y.; Jiang, Y.; Yan, X. P. Probing Mercury Species-DNA Interactions by Capillary Electrophoresis with On-Line Electrothermal Atomic Absorption Spectrometric Detection. Anal. Chem. 2006, 78 (17), 6115-6120.

(34) Lin, Y. H.; Tseng, W. L. Ultrasensitive Sensing of $\mathrm{Hg}^{2+}$ and $\mathrm{CH}_{3} \mathrm{Hg}^{+}$based on the Fluorescence Quenching of Lysozyme Type VIStabilized Gold Nanoclusters. Anal. Chem. 2010, 82 (22), 9194-9200.

(35) Lin, C. Y.; Yu, C. J.; Lin, Y. H.; Tseng, W. L. Colorimetric Sensing of Silver(I) and Mercury(II) Ions Based on an Assembly of Tween 20-Stabilized Gold Nanoparticles. Anal. Chem. 2010, 82 (16), 6830-6837.

(36) Zhang, Z. Y.; Chen, Z. P.; Qu, C. L.; Chen, L. X. Highly Sensitive Visual Detection of Copper Ions Based on the ShapeDependent LSPR Spectroscopy of Gold Nanorods. Langmuir 2014, 30 (12), 3625-3630. 